\title{
Оценка функционального состояния сетчатки у пациентов с диабетическим макулярным отеком с помощью современных методов исследования
}

\section{и их корреляция}

Цель - оценить функциональное состояние сетчатки у пациентов с диабетическим макулярным отеком (ДМО) с помощью современных методов. Объект и методы исследования. Обследовано 20 пациентов, 20 глаз(средний возраст - 57,6士12, 4 года). Всем им диагностирован ДМО. Пациентам, помимо стандартных методов исследования, проводили мультифокальную электроретинографию (мф-ЭРГ), оптическую когерентную томографию (ОКT) и микропериметрию. Результаты. При проведении ОКТ выявлена деформация ретинального профиля. Средняя толщина сетчатки составила 347士10,6 мкм. Установлено, что усредненная плотность биоэлектрического ответа макулярной области сетчатки у пациентов с ДМО резко снижается и составляет 46,37 nV/deg. Средняя светочувствительность, по данным микропериметрии, составила 14, 8 dB. Эти показатели свидетельствуют о снижении как функциональной активности, так и светочувствительности сетчатки. Результаты корреляционного анализа выявили отрицательную корреляцию $(r=-0,442)$ между морфологическими изменениями в центральной зоне сетчатки по данным ОКТ и функциональными показателями - по данным мф-ЭРГ, а также между показателями ОКТ и микропериметрии ( $r=-0,587)$. Между светочувствительностью по данным микропериметрии и функциональной активностью сетчатки - по данным мф-ЭРГ отмечена положительная корреляция ( $r=0,356)$. Выводы. На основании полученных данных можно считать, что при ДМО происходит увеличение толщины сетчатки, что сопровождается депрессией амплитуд мф-ЭРГ и снижением светочувствительности. Выявлена закономерность между морфологическими параметрами фовеальной зоны, ее биоэлектрической активностью и светочувствительностью.

Ключевые слова: диабетическая ретинопатия, мультифокальная электроретинография, оптическая когерентная томография, микропериметрия.

\section{Введение}

Диабетический макулярный отек (ДМО) отмечают у $30 \%$ лиц, болеющих сахарным диабетом (СД) в течение $\geqslant 10$ лет. При своевременной диагностике риск ухудшения зрения у этих пациентов снижается на $50 \%$. Высокая частота развития ДМО у больных СД 2-го типа, получающих инсулинотерапию, обусловлена тяжелым течением основного заболевания, которое сопровождается выраженной артериальной гипертензией и гиперхолестеринемией (Flynn H.W., Smiddy W.E., 2000; American Academy of Ophthalmology, 2017).

Макулярный отек (МО) продолжительное время клинически диагностировали и оценивали путем биомикроофтальмоскопии и флюоресцентной ангиографии - золотых стандартов клинического тестирования для выявления MO (Flynn H.W., Smiddy W.E., 2000; Scholl S. et al., 2010). В настоящее время широкое применение получили технологии точного измерения ретинальной толщины, а также методы, оценивающие функциональную активность и светочувствительность сетчатки и обеспечивающие возможность более раннего скрининга МO, определения его топографии и точного количественного измерения утолщения сетчатки (Early Treatment Diabetic Retinopathy Study Research Group, 1991; Prokofyeva E., Zrenner E., 2012).

Оптическая когерентная томография (ОКТ) - метод оптических измерений (интерферометрия с низкой когерентностью). Информация о структуре и толщине сетчатки обеспечивается путем измерения времени задержки светового эхосигнала, возникающего в результате отражения и рассеивания света различными микроструктурами сетчатки. По времени задержки эхосигнала определяют толщину сетчатки, а по интенсивности отражения - ее структуру. Этот метод сравним с гистологическим, поскольку исследователь имеет возможность получить и проанализировать изображение поперечного среза сетчатки в любой области сканирования (Nagesh B.N. et al., 2016).

Микрофокальная электроретинография (мф-ЭРГ) - математическая модель картирования биоэлектрической активности сетчатки, используемая для исследования функционального состояния, топографии и биоэлектрической активности макулярной области сетчатки. Мф-ЭРГ имеет как практическую, так и научную значимость, позволяет более детально изучить зрительные функции, а также возможные варианты развития диабетических осложнений и их влияние на зрительные функции. Методика безопасна, быстра и объективна, позволяет проводить динамическое наблюдение пациентов и определять доклиническую стадию заболевания, что важно для ранней диагностики (Мансурин Н.Б., Шамшинова А.М., 2009; Bearse M.A., Ozawa G.Y., 2014).

Микропериметрия (МП) представляет собой комбинированное использование компьютерной периметрии и исследования сетчатки, проводимых при помощи фундус-камеры. МП соотносит видимые изменения на глазном дне (анатомию) и дефекты поля зрения (функцию). Это исследование составляет карту светочувствительности макулярной области сетчатки (Midena E., 2006). МП дает возможность провести оценочное исследование зрительной функции пациента, в результате обеспечивается точная корреляция между деталями глазного дна и их светочувствительностью. Также метод позволяет выявить динамику изменения светочувствительности сетчатки в процессе лечения и на момент его начала обозначить степень снижения светочувствительности сетчатки, например в области MO (Midena E., 2006; Grenga P. et al., 2008). 
Цель исследования - изучить топографию, светочувствительность и функциональные изменения в центральной зоне сетчатки, по данным ОКТ, МП и мф-ЭРГ, а также определить их корреляцию у пациентов с ДМО.

\section{Объект и методы исследования}

Проведено обследование 20 пациентов (20 глаз) с СД 2-го типа (инсулиннезависимым) с длительностью заболевания $>10$ лет, среди них 8 женщин и 12 мужчин, возраст $-41-70$ лет (средний возраст - 57,6士12,4 года). Средний уровень гликозилированного гемоглобина составил $8,1 \pm 2,3 \%$. У всех пациентов диагностирован ДМО. Острота зрения была в пределах 0,04-0,6.

Всем пациентам наряду со стандартными методами проводили дополнительные методы исследования: мф-ЭРГ («Retiscan», «Roland Consult», Германия), ОКТ («Carl Zeiss Meditec», США) и МП («MAIA microperimeter», «CenterVue», Италия). Цветное фотографирование выполнено с помощью фундус-камеры «Visucam Pro NM» ("Carl Zeiss Meditec», Германия). С помощью мф-ЭРГ определяли показатели ответа сетчатки на световую стимуляцию (nV) и амплитудные показатели (nV/deg). Стимуляцию центральной зоны сетчатки проводили стимулами гексагональной формы, предъявляемыми псевдослучайным образом. Стимул увеличивался от центра к периферии. Локализация ответа соответствовала каждому гексагональному элементу. В исследовании использовали 103 элемента для более точной локализации патологического процесса и получения ответов с высоким пространственным разрешением. Для изучения характера нарушений в сетчатке биопотенциалы оценивали в 1-, 2-, 3-, 4- и 5-м кольцах стимулируемого поля зрения с радиусами $0-1,9 ; 1,9-6,3 ; 6,3-$ 11,$5 ; 11,5-17,8$ и $17,8-25^{\circ}$ поля зрения соответственно. Математическая модель представляет топографию биоэлектрической активности макулярной области в трехмерном изображении.

При проведении МП у пациентов с ДМО использовали программу «macula-8», покрывающую $8^{\circ}$ центрального поля зрения (поле зрения 0-4웅 точки фиксации) и центрированную на фовеа. В этой программе тестируются 45 точек, в пределах $1^{\circ}$ от точки фиксации они расставлены с частотой $0,5^{\circ}$, вне этой зоны расстояние между точками составляет $1^{\circ}$. Исследование зависит от фиксации пациента и движения глазного яблока, поскольку проецирование производится в соответствии с выбранными ориентирами на глазном дне. Кроме того, МП определяет такие характеристики точки фиксации, как ее локализация и стабильность во времени.

\section{Результаты и их обсуждение}

Основной причиной снижения зрения у больных СД является отек центральной зоны сетчатки.

На глазном дне (рис. 1) отмечены изменения артериальных и венозных сосудов, множественные мелкие внутрисетчаточные кровоизлияния, участки нарушения кровоснабжения, зоны отека, где сетчатка утолщена за счет накопленной жидкости и интраретинальных отложений белково-жировых комплексов (твердых экссудатов). При проведении ОКТ выявлена деформация ретинального профиля, различного размера оптически пустые полости во внутренних и наружных слоях сетчатки, исчезновение центральной ямки, диффузный ретинальный отек. Средняя толщина сетчатки составила $347 \pm 10,6$ мкм.

Результаты проведенного исследования свидетельствуют о том, что усредненная плотность биоэлектрического ответа макулярной области сетчатки у пациентов с ДМО резко снижается. За счет накопленной жидкости происходит снижение функциональной активности биопотенциалов сетчатки во всех 5 кольцах стимулируемого поля зрения и составляет $46,37 \mathrm{nV} / \mathrm{deg}$ (при норме 66,6-130,87 nV/deg).

Центральная светочувствительность, по данным МП, составила $14,8 \mathrm{~dB}$ (при норме 23-25 dB). Эти показатели свидетельствуют о снижении светочувствительности центральной зоны, особенно в местах большего скопления жидкости, и изменении расположения точки фиксации.

Анализ полученных данных подтверждает наличие определенной зависимости между показателями МП сетчатки в фовеа и данными амплитуды мф-ЭРГ в той же зоне у пациентов с МО. Результаты корреляционного анализа выявили умеренную положительную корреляцию между светочувствительностью по дан-

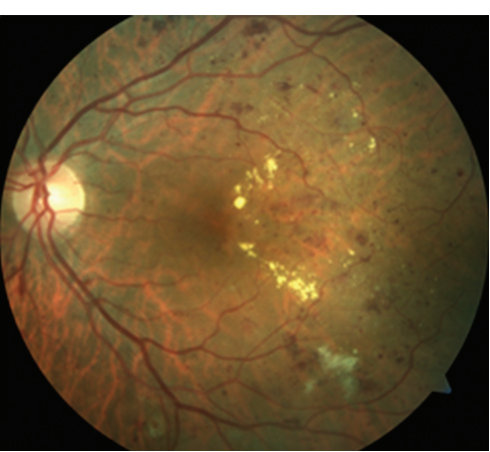

Рис. 1. Фотография глазного дна пациентки М. (61 год) с ДМО. Сетчатка утолщена за счет накопленной жидкости и интраретинальных отложений твердых экссудатов

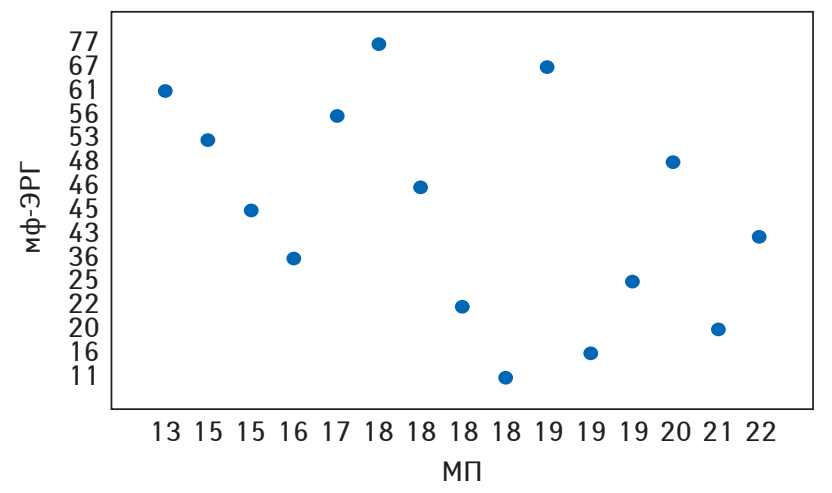

Рис. 2. Показатели данных МП и мф-ЭРГ

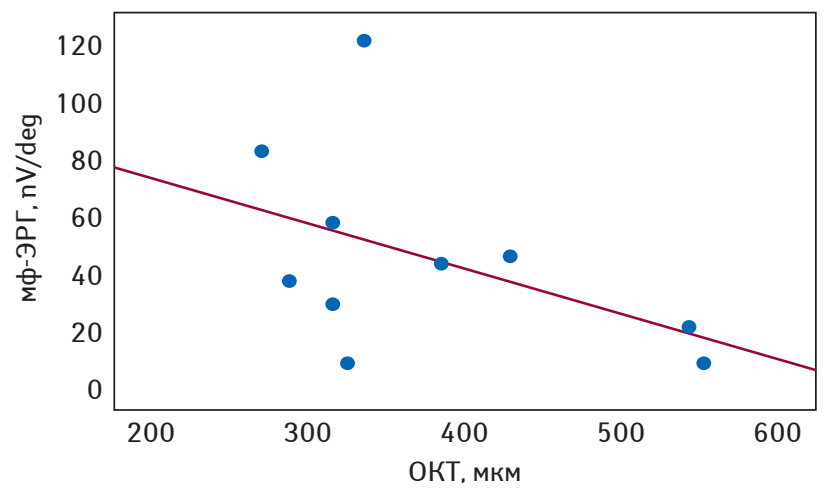

Рис. 3. Соотношение данных ОКТ и мф-ЭРГ

ным МП и функциональной активностью сетчатки - по показателям мф-ЭРГ $(r=0,356)$. Отмечена отрицательная корреляция между показателями ОКТ и МП ( $r=-0,587)$, а также между морфологическими изменениями в центральной зоне сетчатки по данным ОКТ и функциональными показателями - по данным мф-ЭРГ $(r=-0,442)$ (рис. 2).

Средние результаты методов исследования представлены в таблице.

Установлено, что в $80 \%$ случаев причиной резкого снижения зрения у больных СД является специфическое поражение центральной зоны сетчатой оболочки - диабетическая макулопатия (ДМ). Развитие ДМ обусловливают:

- микроокклюзия капилляров (наиболее раннее проявление);

- гиперпроницаемость капилляров, связанная с прорывом внутреннего гематоретинального барьера (стенки капилляров сетчатки), иногда в сочетании с нарушением наружного гематоретинального барьера (пигментного эпителия сетчатки). Общепринято выделение отечной и ишемической форм ДМ. В патогенезе этой патологии установлена роль нарушения наружного и внутреннего гематоретинальных барьеров, влияние медиаторов и тракционного воздействия со стороны стекловидного тела (Early Treatment Diabetic Retinopathy Study Research Group, 1991; Scholl S. et al., 2010). 
Таблица. Средние результаты методов исследования

\begin{tabular}{cccccccc}
\hline Пациент & Пол & $\begin{array}{c}\text { Воз- } \\
\text { раст, } \\
\text { лет }\end{array}$ & Глаз & $\begin{array}{c}\text { Острота } \\
\text { зрения }\end{array}$ & $\begin{array}{c}\text { Толщина } \\
\text { макулы, } \\
\text { мкM }\end{array}$ & $\begin{array}{c}\text { MП, } \\
\mathbf{d B}\end{array}$ & $\begin{array}{c}\text { мф-ЭРГ, } \\
\text { nV/deg }\end{array}$ \\
\hline 1 & Ж & 59 & OD & 0,1 & 421 & 18,2 & 37,65 \\
2 & M & 61 & OD & 0,3 & 347 & 22,3 & 43,41 \\
3 & M & 50 & OS & 0,2 & 387 & 20,8 & 48,36 \\
4 & M & 47 & OD & 0,6 & 398 & 19,5 & 24,88 \\
5 & Ж & 65 & OS & 0,04 & 588 & 11,3 & 36,52 \\
6 & M & 56 & OS & 0,15 & 534 & 18,8 & 47,91 \\
7 & Ж & 45 & OD & 0,4 & 435 & 17,2 & 55,68 \\
8 & Ж & 71 & OD & 0,2 & 447 & 17,7 & 42,11 \\
9 & Ж & 56 & OD & 0,5 & 377 & 21,2 & 44,81 \\
10 & M & 64 & OS & 0,1 & 532 & 17,1 & 15,79 \\
11 & Ж & 58 & OS & 0,3 & 411 & 17,6 & 77,44 \\
12 & K & 58 & OD & 0,05 & 550 & 15,6 & 35,76 \\
13 & Ж & 60 & OD & 0,2 & 467 & 18,2 & 60,78 \\
14 & M & 63 & OD & 0,4 & 483 & 19,4 & 53,26 \\
15 & M & 51 & OD & 0,3 & 396 & 16,7 & 29,71 \\
16 & M & 56 & OD & 0,2 & 467 & 17,1 & 23,4 \\
17 & M & 57 & OS & 0,1 & 522 & 14,3 & 21,8 \\
18 & M & 61 & OD & 0,3 & 388 & 20,4 & 37,5 \\
19 & M & 63 & OD & 0,05 & 564 & 12,3 & 15,6 \\
20 & M & 67 & OS & 0,03 & 547 & 13,4 & 14,8 \\
\hline
\end{tabular}

ОКТ является методом прижизненной диагностики морфологической структуры сетчатки и позволяет с высокой точностью диагностировать МО, оценивать его выраженность количественно, в динамике наблюдать ДМО на фоне проводимого лечения. ОКТ дает возможность оценить толщину сетчатки в мкм, объем в мм $^{3}$ и ее структуру. При наличии ДМО могут отмечаться такие морфологические изменения, как утолщение сетчатки, кистозные изменения, субфовеолярная отслойка нейроэпителия, витреоретинальные тракции, эпиретинальный фиброз, макулярные разрывы (Alkuraya H. et al., 2005). Получение четкого представления о виде МО определяет дальнейшую тактику ведения пациента.

Мф-ЭРГ при диабетической ретинопатии (ДР) изменяется по-разному в зависимости от стадии процесса и степени ишемизации сетчатки, включения в патологический процесс наружных и внутренних слоев сетчатки, фоторецепторов, биполярных и ганглиозных клеток в центральных отделахи на периферии. Так, у пациентов с начальными формами ДР мф-ЭРГ может быть в пределах нормы, однако по мере развития ДР удлиняется межпиковая латентность и латентность до пиков. По данным мф-ЭРГ, даже при начальном фокальном отеке выявляют более глубокие нарушения функции центральной зоны сетчатки, чем это видно офтальмоскопически, а наличие твердых экссудатов приводит кпролонгированию позитивного $\mathrm{P}_{1}$-пика, по сравнению с нормой У пациентов с пролиферативной ДР амплитуда компонентов мфЭРГ значительно снижена (Hood D.C., 2000). Выраженные изменения компонентов м патологическим изменениям в сетчатке, что является плохим прогностическим признаком, и свидетельствуют о функциональной несостоятельности сетчатки. Степень снижения амплитуды b-волны коррелирует с выраженностью МО, а также зависит от наличия добавочных патологических структур (эпиретинальная мембрана, витреоретинальные тракции) (Hood D.C., 2000; Hood D. et al., 2008).

При сопоставлении данных о топографии макулярной области сетчатки и ее биоэлектрической активности, по данным мФ-ЭРГ, различными авторами установлено, что плотность ретинального ответа обратно пропорционально, а латентность компонентов ответа - прямо пропорционально коррелирует с фовеальным утолщением, определяемым на ОКТ. При всех типах ДМО выявлена сильная прямая корреляция между амплитудой b-волны и ретинальной плотностью Р при мф-ЭРГ в области фовеа, а также сильная обратная корреляция этих потенциалов с толщиной фовеальной области сетчатки (Han Y. et al., 2004). Сравнительный анализ мф-ЭРГ и их ассоциации с данными морфологических исследований способствуют повышению эффективности дифференциальной диагностики ДМО.

При ДМО МП позволяет локализовать относительные и центральные скотомы, сопоставить топографию отека с нарушением светочувствительности сетчатки. Наряду с ОКТ МП позволяет детально изучить макулярную зону, особенно при необходимости проведения мониторинга морфологических и функциональных изменений после проведенного лечения (Okada K. et al., 2006).

Детальный анализ светочувствительности показал, что у пациентов с ДМО в фовеа выявлена скотома, по размерам соответствующая или превосходящая зону МО. Установлено, что у пациентов с ДМО имеется систематическая локализация новой точки фиксации в верхнем и верхнем темпоральном секторе макулы, которая не может быть объяснена высокой светочувствительностью или меньшей степенью отека в этих секторах. Предположительно выбор новой точки фиксации взора определяется психофизиологическими особенностями зрительного анализатора (Vujosevic S. et al., 2006).

Выявленные в ходе МП скотомы в месте захвата внутренней пограничной мембраны, а также ишемия сетчатки, впоследствии сменяющаяся истончением ее в макуле и сопровождаемая абсолютной скотомой, заставляют относиться к лечению ДМ с предельной осторожностью. Рекомендуется избегать верхнего и верхнего темпорального сектора при фокальной лазерокоагуляции макулы, поскольку именно там чаще всего располагается функционально важная зона точки фиксации (Okada K. et al., 2006; Vujosevic S. et al., 2006).

Результаты проведенного исследования показали высокую информативность мф-ЭРГ, МП и ОКТ в оценке состояния макулы у пациентов с ДМО. Определено, что подробная характеристика состояния органа зрения у этих пациентов описывается рядом структурных (размеры отека) и функциональных (острота зрения, уровень жалоб, светочувствительность) показателей, некоторые из них взаимосвязаны. Таким образом, обследование пациентов с МО следует проводить только с использованием современных диагностических методик в комплексе.

\section{Выводы}

В ходе работы выявлена корреляция между данными мф-ЭРГ и МП, а также мф-ЭРГ и ОКТ. Показано, что светочувствительность, функциональная активность и топография сетчатки хорошо отражают степень функциональных и морфологических нарушений при ДМО.

\section{Список использованной литературы}

Мансурин Н.Б., Шамшинова А. М. (2009) Локальная и мультифокальная электроретинография в диагностике диабетической ретинопатии. Вестн. офтальмол., 1: 36-39.

Alkuraya H., Kangave D., Abu El-Asrar A.M. (2005) The correlation between optical coherence tomographic features and severity of retinopathy, macular thickness and visual acuity in diabetic macular edema. Int. Ophthalmol., 26: 93-99.

American Academy of Ophthalmology (2017) Diabetic Retinopathy (https://www.aao.org/preferred-practice-pattern/diabetic-retinopathy-ppp-updated-2017)

Bearse M.A., Ozawa G.Y. (2014) Multifocal electroretinography in diabetic retinopathy and diabetic macular edema. Cur. Diab. Rep., 14(9): 526-533.

Early Treatment Diabetic Retinopathy Study Research Group (1991) Classification of diabetic retinopathy from fluorescein angiograms. ETDRS report number 11. Early Treatment Diabetic Retinopathy Study Research Group. Ophthalmology, 98(5 Suppl.): 807-822.

Flynn H.W., Smiddy W.E. (2000) Diabetes and ocular disease: past, present and future therapies: ophthalmology monographs 14. The Foundation of the American Academy of ophthalmology, $334 \mathrm{p}$.

Grenga P., Lupo S., Domanico D. et al. (2008) Efficacy of intravitreal triamcinolone acetonide in long standing diabetic macular edema. A microperimetry and optical coherence study. Retina, 28: 1270-1275.

Han Y., Bearse M.A., Schneck M.E. et al. (2004) Multifocal electroretinogram delays predict sites of subsequent diabetic retinopathy. Invest. Ophthalmol. Vis. Sci., 45: 948-954

Hood D., Bach M., Bridell M. et al. (2008) ISCEV guidelines for clinical multifocal electroretinography - 2007 edition. Doc. Ophthalmol., 116(1): 1-11.

Hood D.C. (2000) Assessing retinal function with the multifocal technique. Prog. Retin. Eye Res., 19(5): 607-646.

Midena E. (2006) Microperimetry. Arch. Soc. Esp. Oftalmol., 81: 183-186.

Nagesh B.N., Takkar B., Azad S., Azad R. (2016) Optical Coherence Tomography and Multifocal Electroretinography in Diabetic Macular Edema: A Neurovascular Relation With Vision. Ophthalmic Surg. Lasers Imaging Retina, 47(7): 626-631.

Okada K., Yamamoto S., Mizunoya S. et al. (2006) Correlation of retinal sensitivity measured with fundus-related microperimetry to visual acuity and retinal thickness in eyes with diabetic macular edema. Eye, Lond., 20: 805-809. 
Prokofyeva E., Zrenner E. (2012) Epidemiology of major eye diseases leading to blindness in Europe: a literature review. Ophthalmic. Res., 47(4): 171-188.

Scholl S., Kirchhof J., Augustin A.J. (2010) Pathophysiology of macular edema. Int. J. Ophthalmol., 224(1): 8-15.

Vujosevic S., Mdena E., Pilotto E. et al. (2006) Diabetic macular edema: correlation between microperimetry and optical coherence tomography findings. Invest. Ophthamol. Vis. Sci., 47: 3044-3051.

\section{Оцінка функціонального стану сітківки у пацієнтів з діабетичним макулярним набряком за допомогою сучасних методів дослідження та їх кореляція \\ М.І. Керімов, Н.А. Шахбазова, У.С. Ісмаїлова}

Резюме. Мета - оцінити функціональний стан сітківки упацієнтів з діабетичним макулярним набряком (ДМН) задопомогою сучаснихметодів Об'єктіметодидослідження. Обстежено 20 пацієнтів, 20 очей(середній вік - 57,6士12,4 року). Усім їм діагностовано ДМН. Пацієнтам, крім стандартних методів дослідження, проводили мультифокальну електроре тинографію (мф-ЕРГ), оптичну когерентну томографію (ОКТ) і мікропериметрію. Результати. При проведенні ОКТ виявлено деформацію ретинального профілю. Середня товщина сітківки становила $347 \pm 10,6$ мкм. Встановлено, що усереднена щільність біоелектричної відповіді макулярної області сітківки у пацієнтів з макулярною набряком різко знижується і становить 46,37 nV/deg. Середня світлочутливість за даними мікропериметрії становила 14,8dB. Ціпоказники свідчать про зниження як функціональної активності, так і світлочутливості сітківки. Результати кореляційного аналізувиявили негативнукореляцію $(r=-0,442)$ між морфологічними змінами в центральній зоні сітківки за даними ОКТ і функціональними показниками - за даними мф-ЕРГ, а також негативну кореляцію між показниками ОКТ і мікропериметрії ( $r=-0,587)$. Між світлочутливістю за даними мікропериметрії та функціональною актив ністю сітківки - за даними мф-ЕРГ відзначено позитивну кореляцію $(r=0,356)$. Висновки. На підставі отриманих даних можна вважати, що при ДМН відбувається збільшення товщини сітківки, що супроводжується депресією амплітуд мф-ЕРГ ізниженням світлочутливості. Виявлено закономірність між морфологічними параметрами фовеальної зони, іiI біоелектричною активністю і світлочутливістю.

Ключові слова: діабетична ретинопатія, мультифокальна електроретинографія, оптична когерентна томографія, мікропериметрія.

\section{Evaluation of the functional state}

of the retina in patients with diabetic macular edema by modern research methods and their correlation

\section{M.I. Kerimov, N.A. Shahbazova, U.S. Ismailova}

Summary. Aim - to evaluate the functional state of the retina in patients with diabetic macular edema (DME) using modern research methods. Materials and methods. We examined 20 patients, 20 eyes(the average age $-57.6 \pm 12.4$ years). All patients were diagnosed with DME. Patients in addition to standard research methods were carried out a multifocal electroretinography (mf-ERG), optical coherence tomography (OCT) and microperimetry. Results. The deformation of the retinal profile was identified on the OCT. The average thickness of the retina was $347 \pm 10.6 \mu \mathrm{m}$. It was established that the average density of the bioelectric response of the macular area of the retina in patients with macular edema is sharply reduced and amounts to $46.37 \mathrm{nV} / \mathrm{deg}$. The average photosensitivity according to the microperimetry was $14.8 \mathrm{~dB}$. These data indicates a decrease of the functional activity and photosensitivity of the retina. The results of the correlation analysis found a negative correlation ( $r=-0.442$ ) between the morphological changes in the centralzone of the retina according to the OCT and functional data according to the $\mathrm{mf}$-ERG as well as a negative correlation between the data of the OCT and microperimetry ( $r=-$ 0.587). It was noted a positive correlation $(r=0.356$ ) between the photosensitivity according to the microperimetry and functional activity of the retina according to the mf-ERG. Conclusions. It can be considered that in patients with DME there is an increase of the thickness of the retina, accompanied by depression of the amplitudes of mf-ERG and decreased photosensitivity. The regularity between the morphological parameters of the foveal zone, its bioelectric activity and photosensitivity was detected.

Key words: diabetic retinopathy, multifocal electroretinography, optical coherence tomography, microperimetry.

\section{Адрес для переписки:}

Шахбазова Нигяр Али кызы

AZ1114, Азербайджан, Баку, ул. Джавадхана, 32/15

Национальный центр офтальмологии имени академика Зарифы Алиевой Министерства здравоохранения Азербайджана

E-mail: rjafarova@bk.ru

Получено 07.07.2017

\section{РЕФЕРАТИВНА ІНФОРМАЦІЯ}

\section{Модуляторы эстрогеновых рецепторов \\ улучшают состояние пациентов \\ с шизофренией}

Ученые из Университетского медицинского центра Утрехта (University Medical Center, Utrecht), Нидерланды, в систематическом обзоре и метаанализе пришли к выводу, что добавление селективного модулятора эстрогеновых рецепторов (СМЭР) ралоксифена кантипсихотической лекарственной терапии может улучшить состояние пациентов с шизофренией. Посколькуэтот препарат может длительное время применяться как женщинами, так и мужчинами, вероятно, он является важным терапевтическим дополнением ксуществующим методам лечения. Результаты исследования представлены онлайн в журнале «NPJ Schizophrenia».

Известно, что существуют гендерные различия в распространенности шизофрении. Так, у мужчин эта патология развивается чаще, чем уженщин, а возраст манифестации у мужчин значительно больше. Уженщин в возрасте 50 лет наблюдается второй пик заболеваемости, которого не бывает у мужчин. Если шизофрения развивается уженщины в периодпременопаузы, протекание заболевания обычно более благоприятно, чем у мужчин-сверстников, с менее выраженными психотическими симптомами, лучшими когнитивными и социальными показателями и меньшим количеством госпитализаций. Специалисты полагают, что такие различия обусловлены тем, что эстрогены могут предотвращать развитие шизофрении. Тем неменее долгосрочное применение гормональных препаратов небезопасно из-за побочных эффектов. СМЭР не обладают побочными эффектами эстрогена и могут длительно приниматься пациентами с шизофренией обоихполов. Наиболее подходящим препаратом для форси- рованной терапии, основанной на протективных способностях эстрогенов, является ралоксифен.

Входе работы авторыпровели количественный систематический обзор 9 рандомизированных контролируемыхисследований, в которых изучалась эффективность ралоксифена по сравнению сплацебо ( $\mathrm{n=561)}$. Оказалось, что прием СМЭР снижал интенсивность симптомов заболевания $(p=0,009)$, какпозитивных $(p=0,02)$, таки негативных $(p=0,02)$, определенных в соответствии со Шкалой позитивных и негативных симптомов (Positive and Negative Syndrome Scale - PANSS). В то же время прием ралоксифена не оказывал значимого влияния на когнитивные функции или вероятность развития депрессии. Дозы принимаемого препарата и продолжительность лечения исследователи не указали. Руководитель работы доктор Жанна де Боер (Janna de Boer) отметила, что входе работы удалось получитьдоказательства того, что ралоксифен является эффективным дополнением к антипсихотическим препаратам, применяемым при шизофрении.

Изучаемая тема очень важна для современной психиатрии, поскольку у большинства пациентов с шизофренией остаются симптомызаболевания, несмотря наприем современныхантипсихотических препаратов. Добавление ралоксифена кстандартной терапии может быть эффективным вспомогательным методом лечения, однако наилучшие результаты следует ожидать у женщин в период пременопаузы.

Boer J., Prikken M., Lei W.U. et al. (2018) The effect of raloxifene augmentation in men and women with a schizophrenia spectrum disorder: a systematic review and meta-analysis. Schizophrenia, Jan. 10 [Epub. ahead of print].

Brooks M. (2018) Hormone therapy may improve schizophrenia outcomes. Medscape, Feb. 22 (https://www.medscape.com/viewarticle/892997).

Юлия Котикович 